\title{
Stability for iterative roots of piecewise monotonic functions
}

\author{
Lin $\mathrm{Li}^{1}$, Wei Song ${ }^{2 *}$ and Yingying Zeng ${ }^{3}$
}

"Correspondence: dawenhxi@126.com

${ }^{2}$ Department of Mathematics, Harbin Institute of Technology, Harbin, Heilongjiang 150080, P.R. China

Full list of author information is available at the end of the article

\begin{abstract}
Global $C^{0}$ and local $C^{1}$ stability of iterative roots for monotonic functions defined on a compact interval, as well as global $C^{1}$ instability under some assumptions, are well-known facts. In this paper, we investigate the stability of iterative roots for piecewise monotonic functions with nonmonotonicity height equal to 1 . We prove the roots are $C^{1}$ locally stable and $C^{0}$ global stable with the same extension.
\end{abstract}

MSC: 39B22; 37E05

Keywords: iterative root; stability; nonmonotonicity height; extension

\section{Introduction}

Given a Banach space $X$ and $r \geq 0, C^{r}(X)$ is defined as the set of all $C^{r}$ self-mappings on $X$. An iterative root of order $k \in \mathbb{N}$ of $F \in C^{r}(X)$ is a function $f \in C^{r}(X)$ that satisfies

$$
f^{k}(x)=F(x), \quad \forall x \in X
$$

where $f^{k}$ denotes the $k$ th iterate of $f$. Being an important problem, iterative roots is connected to the research of embedding flow and topological conjugacy in dynamical systems $[1,2]$, which is also involved in the study of functional equations [3, 4]. To find solutions of equation (1.1) has a long history since 200 years ago [5-9]. In addition to monotonic mappings $[6,7]$, plenty results were obtained for the iterative roots of piecewise monotonic functions [8-12].

Let $I:=[a, b]$ be an interval. A point $x_{0} \in(a, b)$ is referred as a fort of continuous mapping $F: I \rightarrow I$ when $F$ is strictly monotonic in no neighborhood of $x_{0}$. Let $S(F)$ be the set of all forts of $F$. Then $F$ is called a piecewise monotonic function if $N(F):=\# S(f)$ is finite. The set of all such piecewise monotonic self-mappings on $I$ is denoted by $\operatorname{PM}(I, I)$. It is well known that $N(F)$ is nondecreasing under iteration, we define the nonmonotonicity height $H(F)$ of $F$ as the smallest integer $m$ such that $N\left(F^{m}\right)=N\left(F^{m+1}\right)$. When $H(F)=1$, the problem of iterative roots is reduced to be discussed on the characteristic interval (see [8-11]), denoted by $K(F)$. Furthermore, for every continuous iterative root $f$ of $F$ of order $k \geq 2$ there exists a corresponding natural number $\ell(f)$, which maps $I$ into $K(F)$. Such $f$ is called a root of $\ell$-extension (see [11]). The following result gives the iterative roots of those functions with 1-extension.

(c) $2015 \mathrm{Li}$ et al. This article is distributed under the terms of the Creative Commons Attribution 4.0 International License (http://creativecommons.org/licenses/by/4.0/), which permits unrestricted use, distribution, and reproduction in any medium, provided you give appropriate credit to the original author(s) and the source, provide a link to the Creative Commons license, and indicate if changes were made. 
Lemma 1.1 (Theorem 3 in [9]) Suppose $F \in \operatorname{PM}(I, I)$ and $H(F)=1$. Let $K(F)$ be the characteristic interval of $F,[m, M]$ be the range of $F$ and $\left[m^{\prime}, M^{\prime}\right]$ be those of $F$ restricted to $K(F)$. If equation (1.1) has a continuous solution $f_{0}: K(F) \rightarrow K(F)$ maps $[m, M]$ into $\left[m^{\prime}, M^{\prime}\right]$, then there exists a continuous function

$$
f(x):= \begin{cases}f_{0}(x), & x \in K(F), \\ \left.F\right|_{K(F)} ^{-1} \circ f_{0} \circ F(x), & x \in I \backslash K(F),\end{cases}
$$

satisfies $f^{k}(x)=F(x)$ for all $x \in I$.

Clearly, $f$ defined in (1.2) is a root of 1-extension and $K(f)=K(F)$. Conversely, it is easy to prove that all continuous iterative roots of $F$ of order $k$ with 1-extension are in the form of (1.2).

In addition to the study of the existence of iterative roots, more and more attention was paid to their stability. A local result as regards $C^{0}$ stability for a class of strictly monotonic mappings with one fixed point was considered in [13], as well as the global $C^{0}$ stability with more than one fixed point in [14]. Recently, the authors of [15] investigated the $C^{1}$ stability of iterative roots for increasing functions defined on a compact interval. They proved that those iterative roots are $C^{1}$ locally stable but $C^{1}$ globally unstable.

In this paper, we consider the stability of iterative roots for piecewise monotonic functions with nonmonotonicity height equal to 1 . We prove that those roots with the same extension are locally $C^{1}$ stable and globally $C^{0}$ stable.

\section{$2 C^{1}$ stability}

Let $F \in \operatorname{PM}(I, I)$ with $H(F)=1$ and $K(F):=\left[a^{\prime}, b^{\prime}\right] \subset I$ be its characteristic interval. For each $\lambda \in(0,1)$, let

$$
\begin{aligned}
& \mathcal{H}_{-}^{2}(\lambda):=\left\{h \in C^{2}(I): h\left(a^{\prime}\right)=a^{\prime}, h^{\prime}\left(a^{\prime}\right)=\lambda, h^{\prime}(x)>0 \text { and } h(x)<x, \forall x \in\left(a^{\prime}, b^{\prime}\right]\right\}, \\
& \mathcal{H}_{+}^{2}(\lambda):=\left\{h \in C^{2}(I): h\left(b^{\prime}\right)=b^{\prime}, h^{\prime}\left(b^{\prime}\right)=\lambda, h^{\prime}(x)>0 \text { and } h(x)>x, \forall x \in\left[a^{\prime}, b^{\prime}\right)\right\} .
\end{aligned}
$$

For a given integer $k \geq 2$, as discussed in [7], each function $F$ in class $\bigcup_{\lambda \in(0,1)} \mathcal{H}_{-}^{2}(\lambda)$ has a $k$ th order $C^{1}$ iterative root $f$ on $K(F)$, i.e., $f^{k}(x)=F(x)$ for all $x \in K(F)$, which is unique and strictly increasing.

Let the norm $\|\cdot\|_{r}$ be defined by

$$
\|F\|_{r}:=\sup _{x \in I}|F(x)|+\cdots+\sup _{x \in I}\left|F^{r}(x)\right|
$$

for all $r \in \mathbb{N} \cup\{0\}$ and $F \in C^{r}(I)$. Based on the determined formula of $f, C^{1}$ local stability and $C^{1}$ global instability for $f$ were investigated in [15]. The following result shows the stability for those roots.

Lemma 2.1 (Theorem 2.1 in [15]) Let $F \in \mathcal{H}_{-}^{2}(\lambda)\left(\operatorname{or} \mathcal{H}_{+}^{2}(\lambda)\right)$ with a given $\lambda \in(0,1)$ and let $\left(F_{m}\right)$ be a sequence of functions in $\mathcal{H}_{-}^{2}(\lambda)\left(\right.$ or $\left.\mathcal{H}_{+}^{2}(\lambda)\right)$. If

$$
\lim _{m \rightarrow \infty}\left\|F_{m}-F\right\|_{2}=0
$$


then

$$
\lim _{m \rightarrow \infty}\left\|\tilde{f}_{m}-\tilde{f}\right\|_{1}=0
$$

where $\tilde{f}$ and $\tilde{f}_{m}$ are unique kth order $C^{1}$ iterative roots of $F$ and $F_{m}$, respectively, defined on $K(F)$.

Note that a similar result also holds for $F \in \bigcup_{\lambda \in(0,1)} \mathcal{H}_{+}^{2}(\lambda)$.

Let

$$
\mathcal{P} \mathcal{M}_{1}(I, \lambda)_{-}:=\mathcal{P} \mathcal{M}_{1}(I) \cap \mathcal{H}_{-}^{2}(\lambda), \quad \mathcal{P} \mathcal{M}_{1}(I, \lambda)_{+}:=\mathcal{P} \mathcal{M}_{1}(I) \cap \mathcal{H}_{+}^{2}(\lambda)
$$

(see Figures 1 and 2), where

$$
\mathcal{P} \mathcal{M}_{1}(I)=\left\{F \in \operatorname{PM}(I, I): H(F)=1 \text { and } K(F)=\left[a^{\prime}, b^{\prime}\right]\right\}
$$

We first recall some known results. Let $S(F):=\left\{d_{1}, d_{2}, \ldots, d_{N(F)}\right\}$, satisfying $a=d_{0}<d_{1}<$ $\cdots<d_{N(F)}<d_{N(F)+1}=b$. Furthermore, let $I_{i}:=\left[d_{i}, d_{i+1}\right]$ denotes the closure of $i$ th subinterval and $F_{i}:=\left.F\right|_{I_{i}}$. Then $I=\bigcup_{i=0}^{N(F)} I_{i}$ and $F$ is strictly monotone on $I_{i}$.

Figure $1 F \in \mathcal{P M}_{1}(l, \lambda)_{-}$.

Figure $2 F \in \mathcal{P} \mathcal{M}_{1}(I, \lambda)_{+}$.
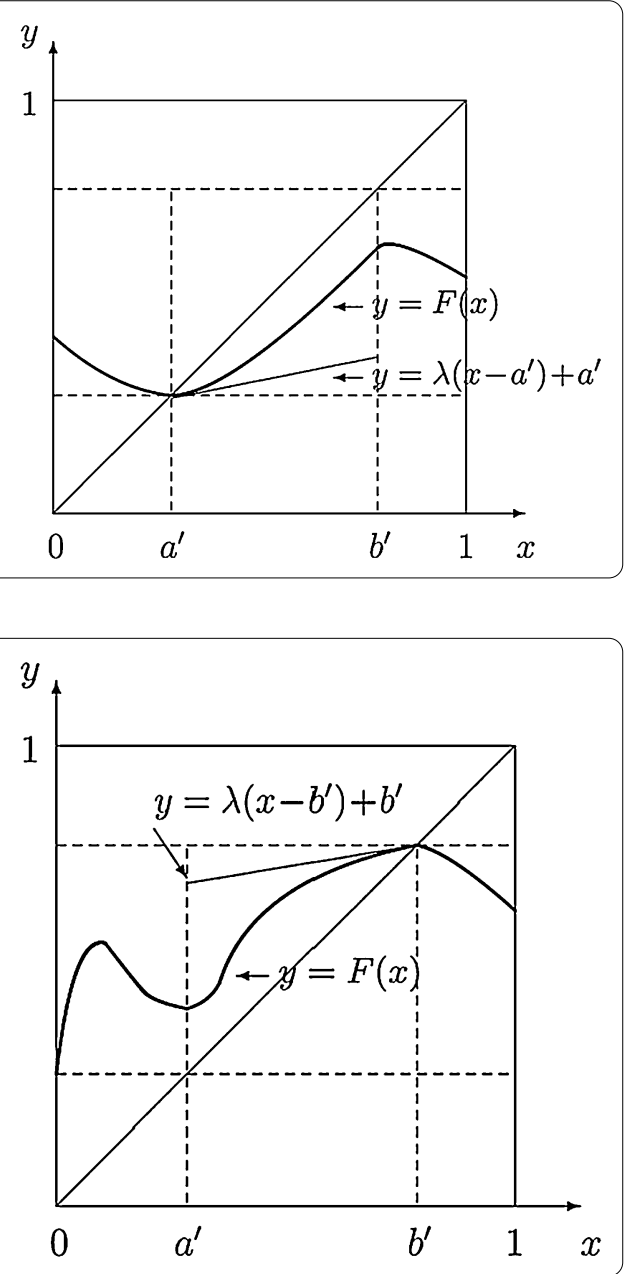
As shown in [11], for each $I_{i}$ there exists a sequence $\left(i_{1}, i_{2}, \ldots, i_{\tau-1}\right)$ in

$$
\{0,1, \ldots, N(F)\}^{\tau-1}:=\underbrace{\{0,1, \ldots, N(F)\} \times \cdots \times\{0,1, \ldots, N(F)\}}_{\tau-1},
$$

where $\tau \leq \min \{k, N(F)\}$ such that

$$
I_{i} \stackrel{f}{\rightarrow} I_{i_{1}} \stackrel{f}{\rightarrow} \cdots \stackrel{f}{\rightarrow} I_{i_{\tau-1}} \stackrel{f}{\rightarrow} K(F)
$$

where $I \stackrel{f}{\rightarrow} J$ denotes $f(I) \subset J$. Then (2.2) gives a correspondence $\tau_{f}:\{0,1, \ldots, N(F)\} \rightarrow$ $\{1, \ldots, \min \{k, N(F)\}\}$ as $i \mapsto \tau$, which is the number that maps $I_{i}$ into the characteristic interval $K(F)$. In [11], the natural number

$$
\ell(f):=\max _{i \in\{0,1, \ldots, N(F)\}} \tau_{f}(i)
$$

is referred to as the pace of the iterative root $f$.

The following theorem is our main result in this section.

Theorem 2.1 Let $F \in \mathcal{P} \mathcal{M}_{1}(I, \lambda)_{-}\left(\right.$or $\left.\mathcal{P} \mathcal{M}_{1}(I, \lambda)_{+}\right)$with some $\lambda \in(0,1)$ and let $\left(F_{m}\right)$ be a sequence of functions in $\mathcal{P} \mathcal{M}_{1}(I, \lambda)_{-}\left(\right.$or $\left.\mathcal{P} \mathcal{M}_{1}(I, \lambda)_{+}\right)$. If

$$
\lim _{m \rightarrow \infty}\left\|F_{m}-F\right\|_{2}=0
$$

then

$$
\lim _{m \rightarrow \infty}\left\|f_{m}-f\right\|_{1}=0
$$

where $f$ and $f_{m}$ are $k$ th order $C^{1}$ iterative roots of $F$ and $F_{m}$ with 1-extension, respectively.

Proof Let $\tilde{F}:=\left.F\right|_{K(F)}$ and $\tilde{F}_{m}:=\left.F_{m}\right|_{K(F)}$, for convenience. It suffices to discuss the case that $F \in \mathcal{P} \mathcal{M}_{1}(I, \lambda)_{-}$since the proof for the case $F \in \mathcal{P} \mathcal{M}_{1}(I, \lambda)_{+}$is similar. We first prove the existence of those iterative roots of $F, F_{m} \in \mathcal{P} \mathcal{M}_{1}(I, \lambda)_{-}$with 1-extension on $I$. As mentioned before, each function $F$ and $F_{m}$ has a $k$ th order $C^{1}$ iterative $\operatorname{root} \tilde{f}$ and $\tilde{f}_{m}$ on their characteristic interval, respectively. Then $\tilde{f}\left(a^{\prime}\right)=\tilde{f}_{m}\left(a^{\prime}\right)=a^{\prime}$ since $a^{\prime}$ is a common fixed point of $F$ and $F_{m}$. Furthermore, by the definition of $\mathcal{H}_{-}^{2}(\lambda)$, we have $\tilde{f}(F([a, b])) \subset$ $\left[F\left(a^{\prime}\right), F\left(b^{\prime}\right)\right]$, which implies that $\tilde{f}$ satisfies the conditions in Lemma 1.1. Hence, $\tilde{f}$ can be extended as a $C^{1}$ iterative root $f$ of $F$ on the whole interval $I$. Similarly, for each $m \in \mathbb{N}$ there exists a continuous iterative $\operatorname{root} f_{m}$ of $F_{m}$, which can be presented by

$$
f_{m}(x):= \begin{cases}\tilde{f}_{m}(x), & x \in K(F), \\ \tilde{F}_{m}^{-1} \circ \tilde{f}_{m} \circ F_{m}(x), & x \in I \backslash K(F),\end{cases}
$$

where $\tilde{f}_{m}^{k}(x)=\tilde{F}_{m}(x)$ for all $x \in K(F)$. Then it follows from Lemma 2.1 that

$$
\lim _{m \rightarrow \infty}\left\|\tilde{f}_{m}-\tilde{f}\right\|_{1}=0
$$


Next, we turn to prove the convergence of $\left(f_{m}\right)$ in $I \backslash K(F)$, by (1.2) and (2.4) we have

$$
\begin{aligned}
\left|f_{m}^{\prime}(x)-f^{\prime}(x)\right|= & \left|\frac{\tilde{f}_{m}^{\prime}\left(F_{m}(x)\right) F_{m}^{\prime}(x)}{\tilde{F}_{m}^{\prime}\left(\tilde{F}_{m}^{-1}\left(\tilde{f}_{m} \circ F_{m}(x)\right)\right)}-\frac{\tilde{f}^{\prime}(F(x)) F^{\prime}(x)}{\tilde{F}^{\prime}\left(\tilde{F}^{-1}(\tilde{f} \circ F(x))\right)}\right| \\
\leq & \left|\frac{\tilde{f}_{m}^{\prime}\left(F_{m}(x)\right) F_{m}^{\prime}(x)}{\tilde{F}_{m}^{\prime}\left(\tilde{F}_{m}^{-1}\left(\tilde{f}_{m} \circ F_{m}(x)\right)\right)}-\frac{\tilde{f}_{m}^{\prime}\left(F_{m}(x)\right) F_{m}^{\prime}(x)}{\tilde{F}^{\prime}\left(\tilde{F}^{-1}(\tilde{f} \circ F(x))\right)}\right| \\
& +\left|\frac{\tilde{f}_{m}^{\prime}\left(F_{m}(x)\right) F_{m}^{\prime}(x)}{\tilde{F}^{\prime}\left(\tilde{F}^{-1}(\tilde{f} \circ F(x))\right)}-\frac{\tilde{f}^{\prime}(F(x)) F^{\prime}(x)}{\tilde{F}^{\prime}\left(\tilde{F}^{-1}(\tilde{f} \circ F(x))\right)}\right| \\
\leq & \left|\frac{\tilde{f}_{m}^{\prime}\left(F_{m}(x)\right) F_{m}^{\prime}(x)}{\tilde{F}_{m}^{\prime}\left(\tilde{F}_{m}^{-1}\left(\tilde{f}_{m} \circ F_{m}(x)\right)\right) \tilde{F}^{\prime}\left(\tilde{F}^{-1}(\tilde{f} \circ F(x))\right)}\right| A(x) \\
& +\left|\frac{1}{\tilde{F}^{\prime}\left(\tilde{F}^{-1}(\tilde{f} \circ F(x))\right)}\right| B(x)
\end{aligned}
$$

for every $x \in I \backslash K(F)$, where

$$
A(x)=\left|\tilde{F}_{m}^{\prime}\left(\tilde{F}_{m}^{-1}\left(\tilde{f}_{m} \circ F_{m}(x)\right)\right)-\tilde{F}^{\prime}\left(\tilde{F}^{-1}(\tilde{f} \circ F(x))\right)\right|
$$

and

$$
B(x)=\left|\tilde{f}_{m}^{\prime}\left(F_{m}(x)\right) F_{m}^{\prime}(x)-\tilde{f}^{\prime}(F(x)) F^{\prime}(x)\right|
$$

In the following, we will estimate the limit of $A(x)$ and $B(x)$. Since

$$
\begin{aligned}
A(x) \leq & \left|\tilde{F}_{m}^{\prime}\left(\tilde{F}_{m}^{-1}\left(\tilde{f}_{m} \circ F_{m}(x)\right)\right)-\tilde{F}^{\prime}\left(\tilde{F}_{m}^{-1}\left(\tilde{f}_{m} \circ F_{m}(x)\right)\right)\right| \\
& +\left|\tilde{F}^{\prime}\left(\tilde{F}_{m}^{-1}\left(\tilde{f}_{m} \circ F_{m}(x)\right)\right)-\tilde{F}^{\prime}\left(\tilde{F}^{-1}(\tilde{f} \circ F(x))\right)\right| \\
\leq & \left\|F_{m}-F\right\|_{2}+\left|\tilde{F}^{\prime}\left(\tilde{F}_{m}^{-1}\left(\tilde{f}_{m} \circ F_{m}(x)\right)\right)-\tilde{F}^{\prime}\left(\tilde{F}_{m}^{-1}\left(\tilde{f} \circ F_{m}(x)\right)\right)\right| \\
& +\left|\tilde{F}^{\prime}\left(\tilde{F}_{m}^{-1}\left(\tilde{f} \circ F_{m}(x)\right)\right)-\tilde{F}^{\prime}\left(\tilde{F}^{-1}\left(\tilde{f} \circ F_{m}(x)\right)\right)\right| \\
& +\left|\tilde{F}^{\prime}\left(\tilde{F}^{-1}\left(\tilde{f} \circ F_{m}(x)\right)\right)-\tilde{F}^{\prime}\left(\tilde{F}^{-1}(\tilde{f} \circ F(x))\right)\right| \\
\leq & \left\|F_{m}-F\right\|_{2}+\left|\tilde{F}^{\prime}\left(\tilde{F}_{m}^{-1}\left(\tilde{f}_{m} \circ F_{m}(x)\right)\right)-\tilde{F}^{\prime}\left(\tilde{F}_{m}^{-1}\left(\tilde{f} \circ F_{m}(x)\right)\right)\right| \\
& +\left|\tilde{F}^{\prime} \circ \tilde{F}^{-1} \circ \tilde{F}_{F} \circ \tilde{F}_{m}^{-1}\left(\tilde{f} \circ F_{m}(x)\right)-\tilde{F}^{\prime} \circ \tilde{F}^{-1} \circ \tilde{F}_{m} \circ \tilde{F}_{m}^{-1}\left(\tilde{f} \circ F_{m}(x)\right)\right| \\
& +\left|\tilde{F}^{\prime}\left(\tilde{F}^{-1}\left(\tilde{f} \circ F_{m}(x)\right)\right)-\tilde{F}^{\prime}\left(\tilde{F}^{-1}(\tilde{f} \circ F(x))\right)\right|
\end{aligned}
$$

by (2.3) and the facts that $\tilde{F}^{\prime} \circ \tilde{F}_{m}^{-1}$ and $\tilde{F}^{\prime} \circ \tilde{F}^{-1}$ are uniformly continuous, we have $A(x) \rightarrow 0$ uniformly in $I$ as $m \rightarrow \infty$.

Moreover, by the definition of $B(x)$, we obtain

$$
\begin{aligned}
B(x) \leq & \left|\tilde{f}_{m}^{\prime}\left(F_{m}(x)\right) F_{m}^{\prime}(x)-\tilde{f}_{m}^{\prime}\left(F_{m}(x)\right) F^{\prime}(x)\right|+\left|\tilde{f}_{m}^{\prime}\left(F_{m}(x)\right) F^{\prime}(x)-\tilde{f}^{\prime}(F(x)) F^{\prime}(x)\right| \\
\leq & \left|\tilde{f}_{m}^{\prime}\left(F_{m}(x)\right)\right|\left|F_{m}^{\prime}(x)-F^{\prime}(x)\right|+\left|F^{\prime}(x)\right|\left|\tilde{f}_{m}^{\prime}\left(F_{m}(x)\right)-\tilde{f}^{\prime}(F(x))\right| \\
\leq & \left|\tilde{f}_{m}^{\prime}\left(F_{m}(x)\right)\right|\left\|F_{m}-F\right\|_{2} \\
& +\left|F^{\prime}(x)\right|\left(\left|\tilde{f}_{m}^{\prime}\left(F_{m}(x)\right)-\tilde{f}^{\prime}\left(F_{m}(x)\right)\right|+\left|\tilde{f}^{\prime}\left(F_{m}(x)\right)-\tilde{f}^{\prime}(F(x))\right|\right) \\
\leq & \left|\tilde{f}_{m}^{\prime}\left(F_{m}(x)\right)\right|\left\|F_{m}-F\right\|_{2}+\left|F^{\prime}(x)\right|\left(\left\|\tilde{f}_{m}-\tilde{f}\right\|_{1}+\left|\tilde{f}^{\prime}\left(F_{m}(x)\right)-\tilde{f}^{\prime}(F(x))\right|\right) .
\end{aligned}
$$


Notice $\tilde{f}$ and $\tilde{f}_{m}$ are $C^{1}$ differentiable. Then it follows from (2.1)-(2.3) that $B(x) \rightarrow 0$ uniformly in $I$ when $m \rightarrow \infty$.

On the other side, note that $\tilde{F}^{\prime}(x), \tilde{F}_{m}^{\prime}(x)>0$ for all $x \in K(F)$, which implies

$$
0<\sup _{x \in I}\left|\frac{1}{\tilde{F}^{\prime}\left(\tilde{F}^{-1}(\tilde{f}(F(x)))\right)}\right|<\infty
$$

and

$$
0 \leq \sup _{x \in I}\left|\frac{\tilde{f}_{m}^{\prime}\left(F_{m}(x)\right) F_{m}^{\prime}(x)}{\tilde{F}_{m}^{\prime}\left(\tilde{F}_{m}^{-1}\left(\tilde{f}_{m}\left(F_{m}(x)\right)\right)\right) \tilde{F}^{\prime}\left(\tilde{F}^{-1}(\tilde{f}(F(x)))\right)}\right|<\infty .
$$

Therefore, in view of (2.6), it gives

$$
\lim _{m \rightarrow \infty}\left\|f_{m}^{\prime}-f^{\prime}\right\|_{0}=0
$$

We conclude $\lim _{m \rightarrow \infty}\left\|f_{m}-f\right\|_{0}=0$. The proof of Theorem 2.1 is completed.

Theorem 2.1 shows the $C^{1}$ stability of iterative roots with 1-extension since the form of roots is determined uniquely by (1.2). Conversely, we conclude the $C^{1}$ instability for those roots with different extensions. Moreover, according to the construction of iterative roots with large extensions (see [11]), we find that the mode of the roots is not unique, which leads to the $C^{1}$ instability for those iterative roots in different modes. Similar to the proof for the 1-extension in Theorem 2.1, we have the following result for larger extensions.

Theorem 2.2 Let $F \in \mathcal{P} \mathcal{M}_{1}(I, \lambda)_{-}\left(\right.$or $\left.\mathcal{P} \mathcal{M}_{1}(I, \lambda)_{+}\right)$with some $\lambda \in(0,1)$ and let $\left(F_{m}\right)$ be a sequence of functions in $\mathcal{P} \mathcal{M}_{1}(I, \lambda)_{-}\left(\right.$or $\left.\mathcal{P} \mathcal{M}_{1}(I, \lambda)_{+}\right)$. If

$$
\lim _{m \rightarrow \infty}\left\|F_{m}-F\right\|_{2}=0
$$

and $F, F_{m}$ has a $k$ th order $C^{1}$ iterative root $f$ and $f_{m}$ with the same mode of extension, then

$$
\lim _{m \rightarrow \infty}\left\|f_{m}-f\right\|_{1}=0
$$

\section{Hyers-Ulam stability}

In this section we prove the Hyers-Ulam stability of equation (1.1).

Suppose $F \in \operatorname{PM}(I, I)$ and $I_{i}$ is an open interval between two consecutive forts (or endpoints) of $F$. Recall $I=\bigcup_{i=0}^{N(F)} \mathrm{cl}\left(I_{i}\right)$ and we let $I(F):=\left\{I_{i}: i=0,1, \ldots, N(F)\right\}$.

Theorem 3.1 Let $F \in \operatorname{PM}(I, I)$ with $H(F)=1$ be given. If the function $f_{s} \in \operatorname{PM}(I, I)$ is Lipschitzian with constants $m>0, M>0$ such that

$$
m|x-y| \leq\left|f_{s}(x)-f_{s}(y)\right| \leq M|x-y|
$$

for every $x, y \in K(F)$, and satisfies:

(A1) $H\left(f_{s}\right)=1$ and $K\left(f_{s}\right)=K(F)$;

(A2) $f_{s}^{k}(x)=F(x)$ for all $x \in K(F)$, and $f_{s}$ maps $K(F)$ onto itself homeomorphically;

(A3) $\left\|f_{s}^{k}-F\right\|_{0} \leq \delta$ for a constant $\delta>0$, 
then equation (1.1) has a solution $f \in \operatorname{PM}(I, I)$ such that

$$
\left\|f_{s}-f\right\|_{0} \leq \frac{1+M}{m^{k}} \delta
$$

Proof This proof is based on the construction of iterative roots of $F \in \operatorname{PM}(I, I)$ with $H(F)=1$. Let

$$
F_{s}(x):=f_{s}^{k}(x), \quad \forall x \in I
$$

It follows from (A1) that $H\left(F_{s}\right)=1$ and $K(F)$ is also the characteristic interval of $F_{s}$ by the iterating mode of $f_{s}$. Thus, from the proof of Theorem 1.1 in [11] and (1.2), each $k$ th order continuous iterative $\operatorname{root} f_{s}$ of $F_{s}$ is extended from that on $K(F)$ by the following formula:

$$
f_{s}(x)=\left.\left.\left.F_{s}\right|_{K(F)} ^{-1} \circ f_{s}\right|_{K(F)} \circ F_{s}\right|_{i}(x), \quad \forall x \in I_{i} \in I(F) \backslash\{K(F)\} .
$$

Hence, the desired function $f \in \operatorname{PM}(I, I)$ can be defined by

$$
f(x):= \begin{cases}f_{s}(x), & x \in K(F), \\ \left.\left.\left.F\right|_{K(F)} ^{-1} \circ f_{s}\right|_{K(F)} \circ F\right|_{I_{i}}(x), & x \in I_{i} \in I(F) \backslash\{K(F)\} .\end{cases}
$$

Since $F_{s}$ maps $K(F)$ onto itself homeomorphically by (A2), it means that $\left.\left.\left.F_{s}\right|_{K(F)} ^{-1} \circ f_{s}\right|_{K(F)} \circ F\right|_{I_{i}}$ is well defined. Since $f_{s}^{k}(x)=F(x)$ for all $x \in K(F)$, one can check that $f$ defined in (3.5) is a solution of equation (1.1).

In order to prove (3.2), it suffices to prove

$$
\left\|f_{s}-f\right\|_{0} \leq \frac{1+M}{m^{k}} \delta, \quad \forall x \in I_{i} \in I(F) .
$$

Obviously, (3.6) holds for every $x \in K(F)$. We next claim that, for every $x \in I_{i}$,

$$
\begin{aligned}
& \text { (K1) }\left.\left.\left|F_{s}\right|_{K(F)}^{-1} \circ f_{s}\right|_{K(F)} \circ F\right|_{I_{i}}(x)-\left.\left.\left.F_{s}\right|_{K(F)} ^{-1} \circ f_{s}\right|_{K(F)} \circ F_{s}\right|_{I_{i}}(x) \mid \leq \frac{M}{m^{k}} \delta, \\
& \left.\left.(\mathrm{K} 2) \quad|F|_{K(F)}^{-1} \circ f_{s}\right|_{K(F)} \circ F\right|_{I_{i}}(x)-\left.\left.\left.F_{s}\right|_{K(F)} ^{-1} \circ f_{s}\right|_{K(F)} \circ F\right|_{I_{i}}(x) \mid \leq \frac{1}{m^{k}} \delta .
\end{aligned}
$$

Actually, it follows from (3.1), (A3), and (3.3) that, for every $x \in I_{i}$,

$$
\left.\left|f_{s}\right|_{K(F)} \circ F\right|_{I_{i}}(x)-\left.\left.\left.f_{s}\right|_{K(F)} \circ F_{s}\right|_{I_{i}}(x)|\leq M| F\right|_{I_{i}}(x)-\left.F_{s}\right|_{I_{i}}(x) \mid \leq M \delta
$$

and

$$
\begin{aligned}
& \left.\left|f_{s}\right|_{K(F)} \circ F\right|_{I_{i}}(x)-\left.\left.f_{s}\right|_{K(F)} \circ F_{s}\right|_{I_{i}}(x) \mid \\
& \quad=\left.\left.\left|F_{s} \circ F_{s}\right|_{K(F)}^{-1} \circ f_{s}\right|_{K(F)} \circ F\right|_{I_{i}}(x)-\left.\left.\left.F_{s} \circ F_{s}\right|_{K(F)} ^{-1} \circ f_{s}\right|_{K(F)} \circ F_{s}\right|_{I_{i}}(x) \mid \\
& \quad \geq\left.\left. m^{k}\left|F_{s}\right|_{K(F)}^{-1} \circ f_{s}\right|_{K(F)} \circ F\right|_{I_{i}}(x)-\left.\left.\left.F_{s}\right|_{K(F)} ^{-1} \circ f_{s}\right|_{K(F)} \circ F_{s}\right|_{I_{i}}(x) \mid,
\end{aligned}
$$

which gives (K1). 
On the other hand, in view of (3.1) and (A3) we have

$$
\begin{aligned}
\delta & \geq\left.\left.\left|F_{s} \circ F\right|_{K(F)}^{-1} \circ f_{s}\right|_{K(F)} \circ F\right|_{I_{i}}(x)-\left.\left.\left.F \circ F\right|_{K(F)} ^{-1} \circ f_{s}\right|_{K(F)} \circ F\right|_{I_{i}}(x) \mid \\
& =\left.\left.\left|F_{s} \circ F\right|_{K(F)}^{-1} \circ f_{s}\right|_{K(F)} \circ F\right|_{I_{i}}(x)-\left.\left.\left.F_{s} \circ F_{s}\right|_{K(F)} ^{-1} \circ f_{s}\right|_{K(F)} \circ F\right|_{I_{i}}(x) \mid \\
& \geq\left.\left. m^{k}|F|_{K(F)}^{-1} \circ f_{s}\right|_{K(F)} \circ F\right|_{I_{i}}(x)-\left.\left.\left.F_{s}\right|_{K(F)} ^{-1} \circ f_{s}\right|_{K(F)} \circ F\right|_{I_{i}}(x) \mid
\end{aligned}
$$

for every $x \in I$ and thus (K2) is proved.

Therefore, consider every $x \in I_{i}$, it follows from (K1) and (K2) that

$$
\begin{aligned}
\left|f_{s}(x)-f(x)\right|= & \left.\left.|F|_{K(F)}^{-1} \circ f_{s}\right|_{K(F)} \circ F\right|_{I_{i}}(x)-\left.\left.\left.F_{s}\right|_{K(F)} ^{-1} \circ f_{s}\right|_{K(F)} \circ F_{s}\right|_{I_{i}}(x) \mid \\
\leq & \left.\left.|F|_{K(F)}^{-1} \circ f_{s}\right|_{K(F)} \circ F\right|_{I_{i}}(x)-\left.\left.\left.F_{s}\right|_{K(F)} ^{-1} \circ f_{s}\right|_{K(F)} \circ F\right|_{I_{i}}(x) \mid \\
& +\left.\left.\left|F_{s}\right|_{K(F)}^{-1} \circ f_{s}\right|_{K(F)} \circ F\right|_{I_{i}}(x)-\left.\left.\left.F_{s}\right|_{K(F)} ^{-1} \circ f_{s}\right|_{K(F)} \circ F_{s}\right|_{I_{i}}(x) \mid \\
\leq & \frac{1+M}{m^{k}} \delta .
\end{aligned}
$$

Thus (3.2) is proved. The proof of Theorem 3.1 is completed.

Theorem 3.2 Let $F \in \operatorname{PM}(I, I)$ with $H(F)=1$ be given. If the function $f_{s} \in \operatorname{PM}(I, I)$ is Lipschitzian with constants $m>0, M>0$ such that

$$
m|x-y| \leq\left|f_{s}(x)-f_{s}(y)\right| \leq M|x-y|
$$

for every $x, y \in K(F)$, and satisfies:

(A1) for each $I_{i} \in I(F)$ there exists a positive integer $\tau \leq \min \{k, N(F)\}$ such that $f_{s}^{\tau}\left(I_{i}\right) \subset K(F)$ and $S\left(f_{s}^{k}\right)=S(F)$

(A2) $f_{s}^{k}(x)=F(x)$ for all $x \in K(F)$, and $f_{s}$ maps $K(F)$ onto itself homeomorphically;

(A3) $\left\|f_{s}^{k}-F\right\|_{0} \leq \delta$ for a constant $\delta>0$,

then equation (1.1) has a solution $f \in \mathrm{PM}(I, I)$ such that

$$
\left\|f_{s}-f\right\|_{0} \leq \frac{1+M}{m^{k}} \delta .
$$

The proof is similar to that of Theorem 3.1.

\section{Examples}

Example 4.1 Consider the mapping $F_{1}:\left[-\frac{1}{2}, 1\right] \rightarrow\left[-\frac{1}{2}, 1\right]$, defined by

$$
F_{1}(x)= \begin{cases}-x^{3}+\frac{1}{2} x^{2}+\frac{1}{4} x, & \forall x \in\left[-\frac{1}{2}, 0\right), \\ \frac{1}{2} x^{2}+\frac{1}{4} x, & \forall x \in[0,1] .\end{cases}
$$

Clearly, $F_{1} \in C^{2}\left(\left[-\frac{1}{2}, 0\right)\right) \cup C^{2}([0,1])$ and $F_{1}$ maps $\left[-\frac{1}{2}, 1\right]$ into $[0,1]$. Thus, $K\left(F_{1}\right)=[0,1]$. Moreover, $\lambda=\frac{1}{4}$ and all conditions in $\mathcal{P} \mathcal{M}_{1}(I, \lambda)_{-}$are satisfied on $K\left(F_{1}\right)$. Therefore, by Theorem 2.1 the $C^{1}$ iterative root of $F_{1}$ with 1 -extension is $C^{1}$ stable.

Example 4.2 Define the mapping $F_{2}:[0,1] \rightarrow[0,1]$ by

$$
F_{2}(x)= \begin{cases}x, & \forall x \in\left[0, \frac{1}{2}\right], \\ 2 x^{2}-2 x+\frac{1}{2}, & \forall x \in\left(\frac{1}{2}, 1\right] .\end{cases}
$$


Obviously, $H\left(F_{2}\right)=1$ and $K\left(F_{2}\right)=\left[0, \frac{1}{2}\right]$. In order to demonstrate the validity of conditions in Theorem 3.1, consider the function $f_{s}:[0,1] \rightarrow[0,1]$ :

$$
f_{s}(x)= \begin{cases}x, & \forall x \in\left[0, \frac{1}{2}\right] \\ -x+1, & \forall x \in\left(\frac{1}{2}, 1\right]\end{cases}
$$

which is Lipschitzian with the constants $m=\frac{49}{50}, M=\frac{51}{50}$ on $K\left(F_{2}\right)$, such that

$$
\frac{49}{50}|x-y| \leq\left|f_{s}(x)-f_{s}(y)\right| \leq \frac{51}{50}|x-y| .
$$

Moreover, one can check that conditions (A1) and (A2) in Theorem 3.1 are true for $f_{s}$. We further calculate that

$$
f_{s}^{2}(x)= \begin{cases}x, & \forall x \in\left[0, \frac{1}{2}\right], \\ -x+1, & \forall x \in\left(\frac{1}{2}, 1\right] .\end{cases}
$$

Hence, it follows that

$$
\left|f_{s}^{2}(x)-F_{2}(x)\right|=\left|2 x^{2}-x-\frac{1}{2}\right| \leq \frac{5}{8}, \quad \forall x \in[0,1] .
$$

Therefore, by Theorem 3.1, equation (1.1) has a solution $f \in \mathrm{PM}([0,1],[0,1])$ such that $\left\|f_{s}-f\right\|_{0} \leq \frac{12,625}{9,604}$ for all $x \in[0,1]$.

\section{Competing interests}

The authors declare that they have no competing interests.

Authors' contributions

The authors contributed equally to the writing of this paper. All authors read and approved the final manuscript.

\section{Author details}

'Department of Mathematics, Physics and Information Engineering, Jiaxing University, Jiaxing, Zhejiang 314001, P.R. China. ${ }^{2}$ Department of Mathematics, Harbin Institute of Technology, Harbin, Heilongjiang 150080, P.R. China.

${ }^{3}$ College of Mathematics and Software Science, Sichuan Normal University, Chengdu, Sichuan 610068, P.R. China.

\section{Acknowledgements}

The authors are grateful to the editor and the referees for their valuable comments. This work is supported by the National Science Foundation of China (Nos. 11301226, 11101105), Zhejiang Provincial Natural Science Foundation of China under Grant No. LQ13A010017 and Scientific Research Fund of Sichuan Provincial Education Department (No. 15ZB0041).

Received: 30 August 2015 Accepted: 1 December 2015 Published online: 15 December 2015

\section{References}

1. Fort, MK Jr:: The embedding of homeomorphisms in flows. Proc. Am. Math. Soc. 6, 960-967 (1955)

2. Shi, Y-G, Li, L, Leśniak, Z: On conjugacy of $r$-modal interval maps with nonmonotonicity height equal to 1. J. Differ. Equ. Appl. 19, 573-584 (2013)

3. Baron, K, Jarczyk, W: Recent results on functional equations in a single variable. Aequ. Math. 61, 1-48 (2001)

4. Targonski, G: Topics in Iteration Theory. Vandenhoeck \& Ruprecht, Göttingen (1981)

5. Babbage, C: An essay towards the calculus of functions. Philos. Trans. R. Soc. Lond. 105, 389-423 (1815)

6. Kuczma, M: Functional Equations in a Single Variable. Polish Sci., Warsaw (1968)

7. Kuczma, M, Choczewski, B, Ger, R: Iterative Functional Equations. Encycl. Math. Appl., vol. 32. Cambridge University Press, Cambridge (1990)

8. Zhang, J, Yang, L: Discussion on iterative roots of piecewise monotone functions. Acta Math. Sin. 26, 398-412 (1983) (in Chinese)

9. Zhang, W: PM functions, their characteristic intervals and iterative roots. Ann. Pol. Math. 65, 119-128 (1997)

10. Li, L, Yang, D, Zhang, W: A note on iterative roots of PM functions. J. Math. Anal. Appl. 341, 1482-1486 (2008)

11. Liu, L, Zhang, W: Non-monotonic iterative roots extended from characteristic intervals. J. Math. Anal. Appl. 378 359-373 (2011) 
12. Liu, L, Jarczyk, W, Li, L, Zhang, W: Iterative roots of piecewise monotonic functions of nonmonotonicity height not less than 2. Nonlinear Anal. 75, 286-303 (2012)

13. Xu, B, Zhang, W: Construction of continuous solutions and stability for the polynomial-like iterative equation. J. Math. Anal. Appl. 325, 1160-1170 (2007)

14. Zhang, W, Zhang, W: Continuity of iteration and stability of iterative roots. J. Comput. Appl. Math. 235, 1232-1244 (2011)

15. Zhang, W, Zeng, Y, Jarczyk, W, Zhang, W: Local $C^{1}$ stability versus global $C^{1}$ unstability for iterative roots. J. Math. Anal. Appl. 386, 75-82 (2012)

Submit your manuscript to a SpringerOpen ${ }^{\circ}$ journal and benefit from:

- Convenient online submission

- Rigorous peer review

- Immediate publication on acceptance

- Open access: articles freely available online

- High visibility within the field

- Retaining the copyright to your article

Submit your next manuscript at $\boldsymbol{s p r i n g e r o p e n . c o m ~}$ 